\title{
hASH1 expression is closely correlated with endocrine phenotype and differentiation extent in pulmonary neuroendocrine tumors
}

\author{
Shi-Xu Jiang ${ }^{1}$, Toru Kameya ${ }^{2}$, Hisao Asamura ${ }^{3}$, Atsuko Umezawa ${ }^{1}$, Yuichi Sato ${ }^{4}$, \\ Jun Shinada ${ }^{5}$, Yasuaki Kawakubo ${ }^{1}$, Toru Igarashi ${ }^{1}$, Kanji Nagai ${ }^{6}$ and Isao Okayasu ${ }^{1}$
}

${ }^{1}$ Department of Pathology, Kitasato University School of Medicine, Sagamihara, Japan; ${ }^{2}$ Pathology Division, Shizuoka Cancer Center Hospital and Research Institute, Shizuoka, Japan; ${ }^{3}$ Department of Chest Surgery, National Cancer Center Hospital, Tokyo, Japan; ${ }^{4}$ Department of Molecular Diagnostics, Kitasato University School of Allied Health Sciences, Sagamihara, Japan; ${ }^{5}$ Department of Chest Surgery, Kitasato University School of Medicine, Sagamihara, Japan and ${ }^{6}$ Department of Chest Surgery, National Cancer Center East Hospital, Kashiwa, Japan

\begin{abstract}
The human homolog 1 of the Drosophila neurogenic achaete-scute genes, hASH1, is specifically expressed in fetal pulmonary neuroendocrine cells and in some neuroendocrine tumor cell lines. However, no data have been gathered regarding its in vivo expression in tumors. hASH1 mRNA expression was investigated by in situ hybridization in 238 surgically resected lung carcinomas, and the correlations between hASH1 expression status and immunostaining results of neuroendocrine markers chromogranin $A$, neural cell adhesion molecule, gastrin-releasing peptide and calcitonin, and clinical outcome were analyzed. $\boldsymbol{h A S H 1}$ expression was detected in $2 / 20(10 \%)$ adenocarcinomas, $4 / 30(13.3 \%)$ typical carcinoids, $11 / 13(84.6 \%)$ atypical carcinoids, $38 / 67(56.7 \%)$ large-cell neuroendocrine carcinomas and $56 / 78(71.8 \%)$ small-cell carcinomas, respectively, but not in any squamous cell carcinoma (0/21) or large-cell carcinoma (0/9). The 2 hASH1+ adenocarcinomas also expressed multiple neuroendocrine markers. Thus, hASH1 expression was restricted to lung cancers with neuroendocrine phenotypes. However, not all neuroendocrine tumors expressed hASH1. Within the entities of large-cell neuroendocrine carcinoma and small-cell carcinoma, hASH1 expression correlated very closely with chromogranin A, gastrin-releasing peptide and calcitonin expression $(P<0.0001, r=0.852)$, but was not related to neural cell adhesion molecule expression $(P=0.8892)$, suggesting that $h A S H 1$ expression, at least in lung cancer, is associated with endocrine phenotype expression other than 'neuroendocrine differentiation' in a broad sense. The fact that $h A S H 1$ was virtually absent in almost fully differentiated typical carcinoids, but was expressed in most, if not all, less differentiated atypical carcinoids as well as large-cell neuroendocrine carcinomas and small-cell carcinomas, suggests that hASH1 expression in lung cancer imitates its early and transient expression in fetal development, and that $h A S H 1$ is instrumental in the establishment, but not in the maintenance, of a cellular endocrine phenotype. Finally, hASH1 expression correlated with a significantly shortened survival in small-cell carcinoma patients $(P=\mathbf{0 . 0 4 1})$.
\end{abstract}

Modern Pathology (2004) 17, 222-229, advance online publication, 5 December 2003; doi:10.1038/modpathol.3800038

Keywords: hASH1; MASH1; achaete; scute; pulmonary neoplasm; neuroendocrine tumor

Based on the absence or presence of cellular neuroendocrine differentiation, lung cancer can be grouped into non-neuroendocrine or neuroendocrine tumors. The former is also called non-smallcell lung cancer and largely comprises squamous

Correspondence: S-X Jiang, Department of Pathology, Kitasato University School of Medicine, Kitasato 1-15-1, Sagamihara, Kanagawa 228-8555, Japan.

E-mail: sxjiang@med.kitasato-u.ac.jp

Received 29 August 2003; revised 22 October 2003; accepted 24 October 2003; published online 5 December 2003 cell carcinoma, adenocarcinoma and large-cell carcinoma. The latter is also a broad entity ranging from low-grade typical carcinoid, intermediategrade atypical carcinoid to the most lethal large-cell neuroendocrine carcinoma and small-cell carcinoma. ${ }^{1-3}$ The neuroendocrine phenotypes may contribute to the virulence of large-cell neuroendocrine carcinoma and small-cell carcinoma via autocrine loops to promote cell growth and protect tumor cells from apoptosis., ${ }^{4,5}$ However, the molecular mechanisms involved in the determination, regulation and maintenance of the neuroendocrine 
phenotypes of lung cancer remain to be further elucidated.

Basic helix-loop-helix (bHLH) proteins form a large family of transcriptional regulatory factors, which are essential in cell fate determination and tissue-specific phenotypic differentiation. ${ }^{6-8}$ Cells of the diffuse neuroendocrine system and neuronal cells share many common biological and morphological features, and they may utilize similar transcriptional mechanisms to determine cell fate and phenotypic differentiation. In Drosophila, the genes of the achaete-scute complex are neurogenic determinants and play a critical role in neuronal commitment and differentiation..$^{9,10}$ Mammalian and human achaete-scute homologs 1, MASH1 and $h A S H 1$, were cloned from a rat sympathetic neuronal precursor cell line and a human medullary thyroid carcinoma cell line, respectively. ${ }^{11,12}$ Subsequent studies have documented that MASH1, like its Drosophila homolog, is specifically and transiently expressed in embryonic neuronal precursors and is instrumental in the development of a subset of neurons. ${ }^{8,13}$ MASH1 is also indispensable for the development of pulmonary neuroendocrine cells, thyroid C cells and adrenal chromaffin cells in fetal mice, and homozygous MASH1 null mice lack pulmonary neuroendocrine cells, while mice deficient in HES1, a specific repressor gene of $h A S H 1$, show a marked increase in pulmonary neuroendocrine cells. ${ }^{14-17}$ In vitro studies also documented restrictive $h A S H 1$ expression in tumor cell lines with neuroendocrine or neuronal differentiation such as pulmonary small-cell carcinoma, medullary thyroid carcinoma and neuroblastoma, ${ }^{12,14,18-23}$ suggesting a key role for $h A S H 1$ in dictating cellular neuroendocrine differentiation in lung cancer. However, few data about in vivo hASH1 expression in lung cancer, as well as in other neuroendocrine tumors, are currently available.

\section{Materials and methods}

\section{Tissue Samples}

Formalin-fixed, paraffin-embedded tissue samples of 238 surgically resected primary lung cancers from the Kitasato University Hospital, Sagamihara; the National Cancer Center Hospital, Tokyo, and the National Cancer Center East Hospital, Kashiwa, Japan, were used. The cases comprised 50 nonsmall-cell lung cancers and 188 neuroendocrine tumors. The former included 21 squamous cell carcinomas, 20 adenocarcinomas and nine large-cell carcinomas, and the latter 30 typical carcinoids, 13 atypical carcinoids, 67 large-cell neuroendocrine carcinomas and 78 small-cell carcinomas. The diagnoses were based on the criteria of the 1999 WHO Histological Typing of Lung and Pleural Tumors. ${ }^{24}$

\section{Nonradioactive mRNA Detection by In Situ Hybridization}

Total RNAs were extracted from LCN1, a large-cell neuroendocrine carcinoma cell line established in our laboratory. A 499-bp $h A S H 1$ fragment corresponding to base pairs 1016-1514 of the hASH1 cDNA sequence ${ }^{12}$ was subcloned into pCR ${ }^{\mathrm{TM}} \mathrm{II}$ (Invitrogen, CA, USA). The digoxigenin-labelled sense and antisense $h A S H 1$ cRNA probes were generated by T7 or SP6 RNA polymerase (Roche Mol. Biochem., Mannheim, Germany), respectively. A modified highly sensitive RNA in situ hybridization method with catalyzed signal amplification based on biotinylated tyramines was employed, which allows messengers to be detected readily with routine formalin-fixed and paraffin-embedded samples. $^{25}$ In brief, after hybridization with sense or antisense cRNA $h A S H 1$ probe at $50^{\circ} \mathrm{C}$ for $2 \frac{1}{2} \mathrm{~h}$, the sections were treated with ribonuclease and washed under highly stringent conditions followed by endogenous biotin blocking with a Biotin Blocking System (DAKO, CA, USA). Then, the sections were sequentially incubated with horseradish peroxidase conjugated anti-digoxigenin antibody (DAKO, Denmark), biotinylated tyramide and horseradish peroxidase-labelled streptavidin of the GenPoint kit (DAKO, CA, USA). Hybridization results were visualized with 3,3'-diaminobenzidine and the slides were counterstained with hematoxylin.

A nude mouse xenograft of the LCN1 in which constitutive $h A S H 1$ expression had been confirmed by RT-PCR was used as a positive sample control. Hybridization of a 340-bp antisense beta-2-microglobulin cRNA probe, when necessary, was also used as an internal control to confirm the quality of the messengers. The negative controls included usage of a sense probe and treatment of the antisense probe or sections with ribonuclease. Furthermore, a squamous cell carcinoma without detectable hASH1 expression by RT-PCR was also used as a negative sample control.

The results were scored as negative, or focally, patchily or diffusely positive when definite hybridization signals were detected in none, less than 10 , $10-50 \%$ or more than $50 \%$ of the tumor cells in individual tumors, respectively. The positive hybridization signals were also scored as mild, moderate or intense, according to intensity.

\section{Immunohistochemical Staining}

Immunohistochemical staining of general neuroendocrine markers, chromogranin A $(1000 \times$ dilution, Dako, Denmark) and neural cell adhesion molecule $(200 \times$ dilution, Nippon Kayaku, Tokyo, Japan), and peptide hormones, gastrin-releasing peptide $(2000 \times$ dilution, Dako, Japan) and calcitonin $(200 \times$ dilution, Incstar, Stillwater, MN, USA), were performed with the standard labelled streptavidinbiotin method, as described previously.,26 


\section{Statistical Analysis}

Correlation of categorical variables between different groups was assessed using Fisher's exact test, Mann-Whitney $U$-test or the $\chi^{2}$-test. Comparison of survival rates was performed using Kaplan-Meier survival rate curves analyzed with the log-rank test. A probability value $(P)$ less than or equal to 0.05 was considered significant. All analyses were performed with StatView statistical software, version 5.0.

\section{Results}

\section{hASH1 Expression in Non-small-cell Lung Cancer}

No hASH1 hybridization signals could be detected in all of the 21 squamous cell carcinomas and nine large-cell carcinomas. Two (10\%) of the 20 adeno- carcinomas expressed $h A S H 1$ and both showed patchy and moderate hybridization signals.

\section{hASH1 Expression in Carcinoid Tumors}

Of the 30 typical carcinoids, only four (13.3\%). cases showed hASH1 expression, with a diffuse pattern in two and a focal pattern in another two cases (Figure 1a). One was intense, while the other three revealed weak hybridization signals. On the other hand, $11(84.6 \%)$ of the 13 atypical carcinoids were $\mathrm{hASH1}^{+}$and each of them demonstrated hybridization signals in a diffuse pattern (Figure 1b). The hybridization signals were intense in three, moderate in seven and weak in one case, respectively. There was a significant difference in $h A S H 1$ expression incidence between typical carcinoids
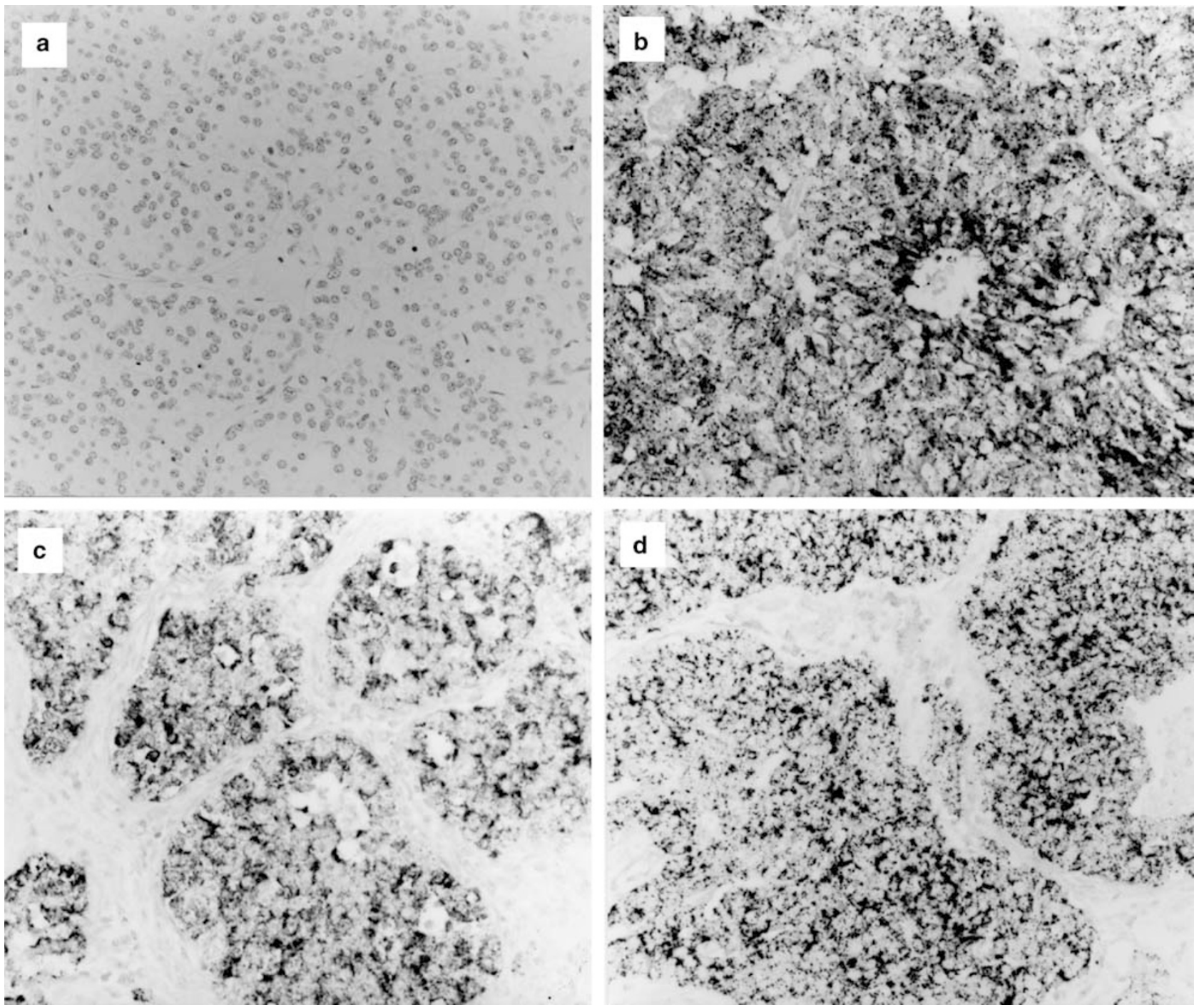

Figure 1 Representative hASH1 mRNA in situ hybridization results in pulmonary neuroendocrine tumors. No hASH1 hybridization signals can be detected in typical carcinoid (a), while atypical carcinoid (b), large-cell neuroendocrine carcinoma (c) and small-cell carcinoma (d) are strongly and diffusely positive for $h A S H 1$. 
and atypical carcinoids (Fisher's exact test, $P<0.0001)$.

\section{hASH1 Expression in Large-cell Neuroendocrine Carcinoma and Small-cell Carcinoma}

Of the 67 large-cell neuroendocrine carcinomas, 38 $(56.7 \%)$ cases showed readily detectable $h A S H 1$ hybridization signals. A total of 30 cases showed diffuse staining, while only six and two cases showed patchy and focal staining, respectively. The hybridization signals were intense in 16, moderate in 13 and weak in nine cases (Figure 1c).

hASH1 hybridization signals were observed in 56 $(71.8 \%)$ of the 78 small-cell carcinomas; diffuse in 49 , patchy in five and focal in two cases. The hybridization signals were intense in 21 , moderate in 23 and weak in 12 cases, respectively (Figure 1d).

There was no statistical difference in hASH1 expression incidence between large-cell neuroendocrine carcinoma and small-cell carcinoma, although $h A S H 1$ mRNA was detected in a higher percentage of the latter (Fisher's exact test, $P=0.0807$ ). The same was true for the distribution range and intensity of $h A S H 1$ hybridization signals between large-cell neuroendocrine carcinoma and small-cell carcinoma (Mann-Whitney $U$-test, $P=0.0549$ and 0.1417 , respectively).

Of the 67 large-cell neuroendocrine carcinomas and 78 small-cell carcinomas, eight and 11 cases were combined tumors, ${ }^{24}$ respectively, in which an element of squamous cell carcinoma or adenocarcinoma coexisted. Of these 19 combined carcinomas, 10 expressed $h A S H 1$, and the hybridization signals were strictly restricted to the large-cell neuroendocrine carcinoma and small-cell carcinoma regions, while the squamous cell carcinoma and adenocarcinoma elements were completely negative for $h A S H 1$ (Figure 2).

The LCN1 xenograft, the positive sample control, demonstrated intense and diffuse $h A S H 1$ hybridization signals. No signals could be observed in the squamous cell carcinoma, the negative sample control, or when a sense probe or ribonuclease pretreated antisense probe or sections were used.
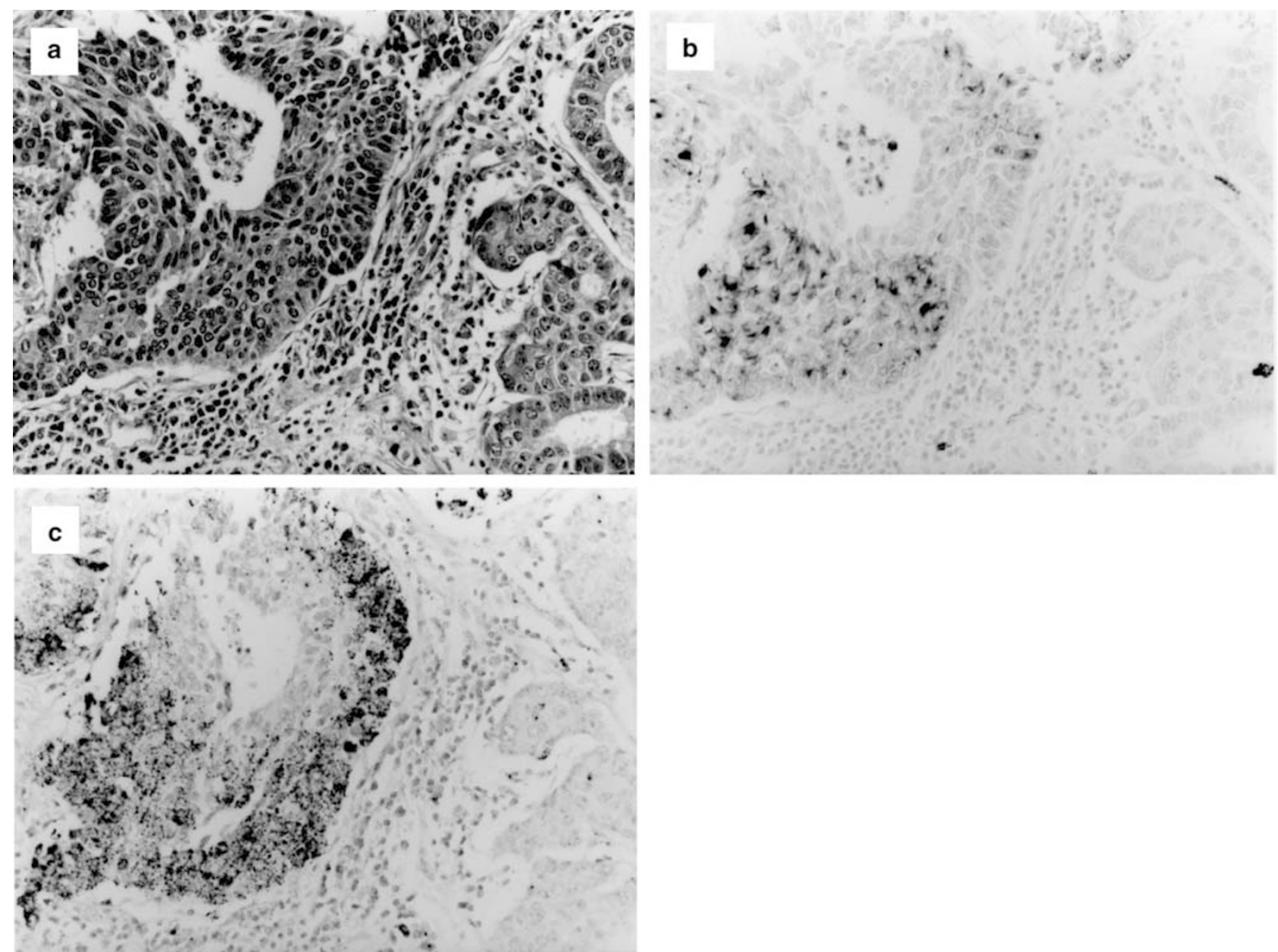

Figure 2 (a) A combined large-cell neuroendocrine carcinoma (left) and adenocarcinoma (right). (b) Only the large-cell neuroendocrine carcinoma element is positive for chromogranin A (left). (c) On a consecutive section, the hASH1 hybridization signals are also confined to the large-cell neuroendocrine carcinoma element. 
All the $h A S H 1^{-}$tumors showed beta-2-microglobulin hybridization signals, indicating that the lack of $h A S H 1$ signals in these specimens was not due to degraded tumor mRNA.

\section{Immunohistochemical Staining Results}

As mentioned above, two adenocarcinomas showed patchily moderate $h A S H 1$ hybridization signals. Also, in the same areas of these two tumors, positive staining for chromogranin A and calcitonin was observed on consecutive sections, and one of them was also positive for gastrin-releasing peptide. The remaining $18 \mathrm{hASH}^{-}$adenocarcinomas, and all the 21 squamous cell carcinomas and nine large-cell carcinomas, were completely negative for the general neuroendocrine markers or peptide hormones.

Of the 43 typical carcinoids and atypical carcinoids, all and 38 cases were positive for chromogranin A and neural cell adhesion molecule, respectively, and 32 and 17 cases were positive for gastrin-releasing peptide and calcitonin, respectively, with all 17 calcitonin $^{+}$cases being also positive for gastrin-releasing peptide.

Of the 145 large-cell neuroendocrine carcinomas and small-cell carcinomas, 88 and 133 were positive for chromogranin $A$ and neural cell adhesion molecule, respectively, with all cases expressing at least one marker and 80 cases being positive for both the markers, confirming their neuroendocrine differentiation. In all, 95 and 51 tumors also expressed gastrin-releasing peptide and calcitonin, respectively, with 45 cases expressing both the peptide hormones. When compared with hASH1 expression status, an obvious tendency for $h A S H 1$ expression in those tumors expressing chromogranin A was found. 79/94 $\mathrm{hASH}^{+}$large-cell neuroendocrine carcinomas and small-cell carcinomas were positive, while the majority $(42 / 51)$ of $h A S H 1^{-}$tumors were negative for chromogranin $\mathrm{A}$; and there was a close positive correlation between $h A S H 1$ and chromogranin A expressions $\left(\chi^{2}\right.$-test, $P<0.0001$, $r=0.649$ ). On the other hand, $h A S H 1$ expression showed no relationship to neural cell adhesion molecule expression status (Table 1, $\chi^{2}$-test, $P=0.8892$ ).

Chromogranin A is a constituent of secretory granules of normal as well as neoplastic neuroendocrine cells, and its expression is closely associated with the cellular endocrine phenotype defined as peptide hormone/biogenic amine production, storage and secretion. Thus, the correlation between $h A S H 1$ expression and gastrin-releasing peptide or calcitonin expression was also analyzed. In all, 86 and 49 of the $94 \mathrm{hASH}^{+}$large-cell neuroendocrine carcinomas and small-cell carcinomas were positive for gastrin-releasing peptide and calcitonin, respectively, while 42 and 49 of the $51 \mathrm{hASH}^{-}$tumors were negative for gastrin-releasing peptide and calcitonin, respectively. Thus, a positive correlation
Table 1 Irrelative $h A S H 1$ and neural cell adhesion molecule expression in 145 large-cell neuroendocrine carcinomas and small-cell carcinomas

\begin{tabular}{lcc}
\hline & hASH1 positive & hASH1 negative \\
\hline $\begin{array}{l}\text { Neural cell adhesion } \\
\text { molecule positive }\end{array}$ & 86 & 47 \\
$\begin{array}{l}\text { Neural cell adhesion } \\
\text { molecule negative }\end{array}$ & \multicolumn{2}{c}{$P<0.8892, r=-0.012$} \\
& & 4 \\
\hline
\end{tabular}

Table 2 hASH1 and chromogranin A/gastrin-releasing peptide/ calcitonin (CGA/GRP/CT) expression in 145 large-cell neuroendocrine carcinomas and small-cell carcinomas

\begin{tabular}{|c|c|c|}
\hline & hASH1 positive & hASH1 negative \\
\hline \multirow{2}{*}{$\begin{array}{l}\text { Any of CGA/GRP/CT } \\
\text { positive }\end{array}$} & 94 & 10 \\
\hline & \multicolumn{2}{|c|}{$P<0.0001, r=0.852$} \\
\hline $\begin{array}{l}\text { All of CGA/GRP/CT } \\
\text { negative }\end{array}$ & 0 & 41 \\
\hline
\end{tabular}

between $h A S H 1$ expression and gastrin-releasing peptide or calcitonin expression was also found $\left(\chi^{2}-\right.$ test, $P<0.0001, r=0.742$ and $r=0.482$, respectively). Furthermore, when the immunostaining results were taken together, as shown in Table 2, all the $94 \mathrm{hASH}^{+}$large-cell neuroendocrine carcinomas and small-cell carcinomas expressed chromogranin A and/or gastrin-releasing peptide and/or calcitonin, while the vast majority of $h A S H 1^{-}$ tumors were negative for these neuroendocrine markers; and there was a very close positive correlation between hASH1 expression and chromogranin A/gastrin-releasing peptide/calcitonin expression ( $\chi^{2}$-test, $\left.P<0.0001, r=0.852\right)$.

\section{Clinical Significance of $h A S H 1$ Expression in Pulmonary Neuroendocrine Tumors}

Complete follow-up information was available for all cases except for one atypical carcinoid and four small-cell carcinoma patients. The Kaplan-Meier survival curves of the patients are shown in Figure 3a. There was a significant difference in overall survival for typical carcinoid patients vs atypical carcinoid patients and for atypical carcinoid patients vs large-cell neuroendocrine carcinoma patients (log-rank test, $P=0.0259$ and 0.0183 , respectively), but not for large-cell neuroendocrine carcinoma patients vs small-cell carcinoma patients $(P=0.4692)$. One of the four $h A S H 1^{+}$typical carcinoid patients died of the disease, while all 26 $h A S H 1^{-}$typical carcinoid patients survived. Two of the $10 h A S H 1^{+}$and one of the two $h A S H 1^{-}$atypical carcinoid patients succumbed to the disease, 

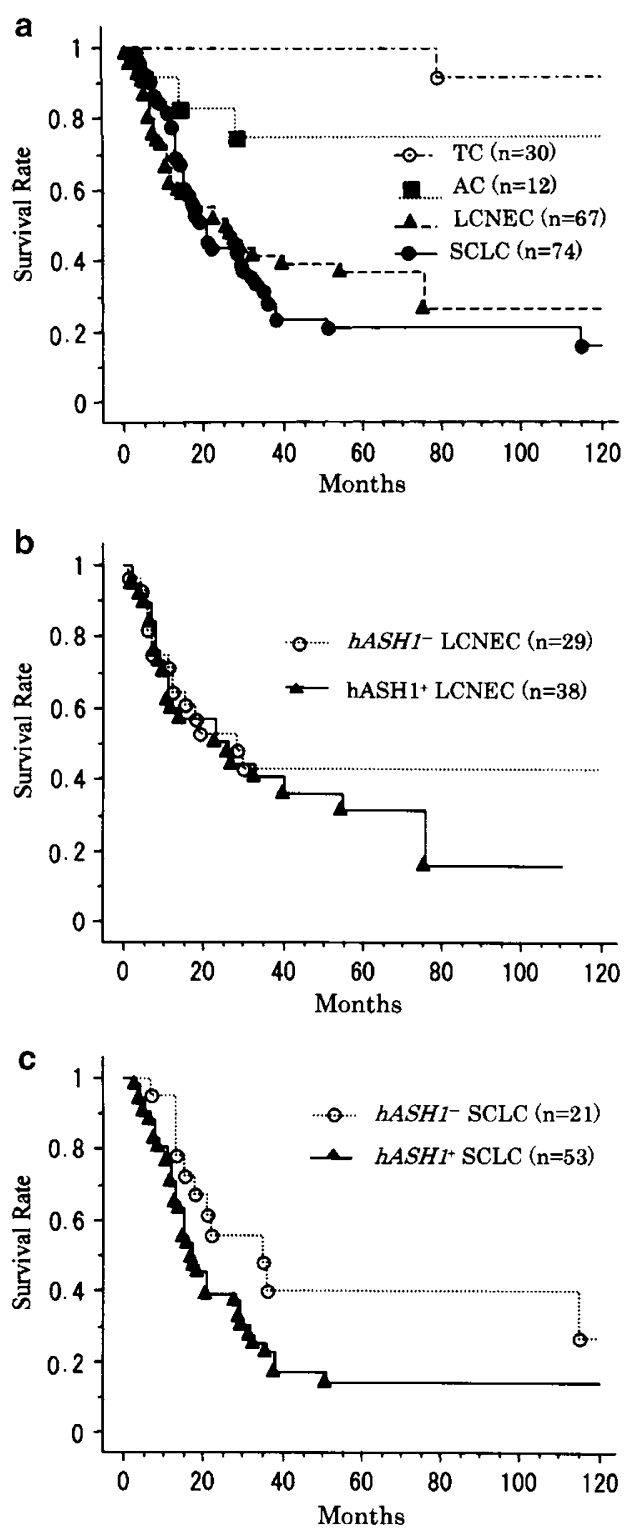

Figure 3 (a) Kaplan-Meier survival curves and log-rank test for patients with typical carcinoid (TC), atypical carcinoid (AC), large-cell neuroendocrine carcinoma (LCNEC) and small-cell carcinoma (SCLC). There was a significant difference in overall survival for typical carcinoid vs atypical carcinoid and for atypical carcinoid vs large-cell neuroendocrine carcinoma ( $P=0.0259$ and $P<0.0183$, respectively), but not for large-cell neuroendocrine carcinoma vs small-cell carcinoma $(P=0.4692)$. (b) Kaplan-Meier survival curves and log-rank test for large-cell neuroendocrine carcinoma patients by $h A S H 1$ expression status $(P=0.4702)$. (c) Kaplan-Meier survival curves and log-rank test for small-cell carcinoma patients by $h A S H 1$ expression status $(P=0.041)$.

respectively. The small numbers of $h A S H 1^{+}$typical carcinoid and $\mathrm{hASH1}^{-}$atypical carcinoid cases impeded statistical analysis for survival difference based on $h A S H 1$ expression status. The 2 - and 5year overall survival rates for the $38 \mathrm{hASH} 1^{+}$and the $29 \mathrm{hASH}^{-}$large-cell neuroendocrine carcinoma patients were 51.0 and $31.5 \%$, and 51.9 and $42.1 \%$ respectively. Although $h A S H 1^{+}$large cell neuro- endocrine cases had a shorter 5-year survival than $h A S H 1^{-}$patients, the difference was not significant (Figure 3b, log-rank test, $P=0.4702$ ). However, hASH1 expression was associated with a worse prognosis in small-cell carcinoma patients. As shown in Figure 3c, the 2- and 5-year survival for patients with $h A S H 1^{+}$and $h A S H 1^{-}$small-cell carcinoma are 39.0 and $14.6 \%$, and 55.9 and $40.0 \%$, respectively, and there was a significant difference in prognosis (log-rank test, $P=0.041$ ).

\section{Discussion}

It is important to define, at a molecular level, the mechanisms involved in regulating neuroendocrine differentiation of lung cancers because this may contribute to the virulence of small-cell carcinoma and large-cell neuroendocrine carcinoma. ${ }^{4,5}$ In the present study, hASH1 expression was detected in 4/30 typical carcinoids, 11/13 atypical carcinoids, 38/67 large-cell neuroendocrine carcinomas and 56/78 small-cell carcinomas. Of the 50 non-smallcell lung cancers, only two adenocarcinomas expressed $h A S H 1$ in a patchy pattern. However, both of these two $h A S H 1^{+}$adenocarcinomas also expressed neuroendocrine markers. Thus, our data confirmed and extended the findings of previous in vitro studies that $h A S H 1$ was specifically expressed in lung cancers with neuroendocrine differentiation, ${ }^{12,14,18,19}$ indicating that besides being instrumental in development of a subset of neurons and neuroendocrine cells, ${ }^{8,13-17}$ hASH1 also plays a key role in regulating neuroendocrine differentiation of tumor cells.

The term 'neuroendocrine differentiation' in a broad sense is used to define cells expressing a neural and/or an endocrine phenotype. The markers of the former include cytosolic and cell membrane markers common to neurons and endocrine cells such as neuron-specific enolase, neuron-specific class III beta-tubulin and neural cell adhesion molecule. On the other hand, an endocrine phenotype should be defined as the capacity of a given cell to produce and secrete peptide hormone(s)/biogenic amine(s). Chromogranins, a group of acidic monomeric proteins, are regarded as markers of the endocrine phenotype, as they are constituents of the matrix of secretory granules where peptides/ amines are stored. ${ }^{27-29}$ It is well known that some pulmonary neuroendocrine tumors express neural marker(s), but are completely negative for either chromogranin A or hormones, indicating these tumors have neural differentiation, but lack an endocrine phenotype. In the present study, most $h A S H 1^{+}$large-cell neuroendocrine carcinomas and small-cell carcinomas expressed chromogranin A, while the vast majority of $h A S H 1^{-}$large-cell neuroendocrine carcinomas and small-cell carcinomas were completely negative for chromogranin A $(P<0.0001)$. Furthermore, hASH1 expression was 
completely irrelative to neural cell adhesion molecule expression $(P=0.8892)$. Thus, we suspected that a linkage existed between $h A S H 1$ expression and cellular endocrine phenotype other than 'neuroendocrine differentiation' in a broad sense.

However, 15 hASH1 $^{+}$large-cell neuroendocrine carcinomas and small-cell carcinomas showed no chromogranin A expression. Although chromogranin A expression generally parallels hormone expression, it is not uncommon for pulmonary and other neuroendocrine tumors to express hormone(s), but be negative for chromogranin A. One possible explanation for this is that chromogranins $\mathrm{B}$ and/or $\mathrm{C}$, but not chromogranin $\mathrm{A}$, are alternatively present in these tumors. ${ }^{30}$ According to our previous studies, ${ }^{3,26}$ gastrin-releasing peptide and calcitonin are the most frequently expressed hormones in lung cancer, and a combination of immunostainings of these two hormones and chromogranin A can discriminate most, if not all, lung cancers with an endocrine phenotype. In the present study, first, either gastrin-releasing peptide or calcitonin expression was related to $h A S H 1$ expression in large-cell neuroendocrine carcinomas and small-cell carcinomas $(P<0.0001$, respectively). Second, when taking the staining results together, there was a very close positive correlation between $h A S H 1$ and chromogranin A/gastrin-releasing peptide/calcitonin expressions $(P<0.0001, r=0.852)$. Thus, the present results based on a substantial number of cases strongly suggested that $h A S H 1$ expression, at least in the context of lung cancer, is associated with a cellular endocrine phenotype. This notion is supported by a recent observation that development of most adrenal medullary cells in MASH1-deficient mice were arrested at an early stage, and the medullary cells expressed high levels of neurofilament and resembled immature sympathetic neuroblast, but failed to acquire an endocrine phenotype. ${ }^{17}$

On the other hand, hASH1 expression was virtually absent in typical carcinoid, which has the most overt and mature endocrine phenotype. Across the spectrum of pulmonary neuroendocrine tumors, typical carcinoid and small-cell carcinoma represent the two ends of the differentiation extent, with atypical carcinoid and large-cell neuroendocrine carcinoma in between. The Drosophila and mammalian homologs of $h A S H 1$ are only transiently expressed in neuronal progenitor cells and pulmonary neuroendocrine cells during early fetal development, ${ }^{8,9,11,13}$ and in vitro hASH1 expression preceded and overlapped induced neuronal differentiation of neuroblastoma and P19 cells, but vanished before the overt neuronal phenotype appeared. $^{22,31}$ Silencing of $h A S H 1$ expression also parallelled the development of induced mature $C$ cell phenotype of medullary thyroid carcinoma cells. ${ }^{20}$ In the present study, $h A S H 1$ expression also depended on differentiation extent, and was detected in the vast majority of less differentiated atypical carcinoids, as well as in large-cell neuroendocrine carcinomas and small-cell carcinomas, but was virtually absent in almost fully differentiated typical carcinoids. These data together strongly suggest that $h A S H 1$ expression in pulmonary neuroendocrine tumors imitates its early and transient expression pattern during development, and $h A S H 1$ is instrumental in establishment of a cellular endocrine phenotype, but is not necessary for maintenance of the fully developed endocrine phenotype. Thus, it is not surprising that clinically benign pheochromocytomas showed no hASH1 expression, while neural crest-derived malignant tumors expressed $h A S H 1 .{ }^{12,20,22,23}$ Our typical carcinoids and atypical carcinoids were differentially diagnosed based on mitotic count and/or presence or absence of necrosis. ${ }^{2,24}$ As one of the four $h A S H 1^{+}$typical carcinoid patients died of the disease while all $26 h A S H 1^{-}$typical carcinoid patients survived, $h A S H 1^{+}$typical carcinoid diagnosed with these criteria might be biologically more like an atypical carcinoid.

hASH1 expression was associated with a significantly shortened overall survival in small-cell carcinoma patients, suggesting the potential prognostic significance of $h A S H 1$. Since peptide hormones such as gastrin-releasing peptide can promote cell growth and protect tumor cells from apoptosis through autocrine loops, ${ }^{4,5}$ hASH1 might confer a role on the biological behavior of small-cell carcinoma via its impact on the endocrine phenotype of the tumor cells. However, hASH1 expression was not significantly associated with clinical outcome in large-cell neuroendocrine carcinoma patients, although a shorter 5-year survival was observed in patients with $h A S H 1^{+}$tumor. The prognostic significance of $h A S H 1$ expression in pulmonary neuroendocrine tumors remains to be clarified.

\section{Acknowledgements}

This work was supported by Grants from the Ministry of Education, Culture, Sports, Science and Technology (15590314 and High-tech research center) and from the Ministry of Health, Labor and Welfare (11-19, 2002), Japan

\section{References}

1 Travis WD, Linnoila RI, Tsokos MG, et al. Neuroendocrine tumors of the lung with proposed criteria for large-cell neuroendocrine carcinoma. An ultrastructural, immunohistochemical, and flow cytometric study of 35 cases. Am J Surg Pathol 1991;15:529-553.

2 Travis WD, Rush W, Flieder DB, et al. Survival analysis of 200 pulmonary neuroendocrine tumors with clarification of criteria for atypical carcinoid and its separation from typical carcinoid. Am J Surg Pathol 1998;22:934-944. 
3 Jiang SX, Kameya T, Shoji $\mathrm{M}$, et al. Large cell neuroendocrine carcinoma of the lung. A histologic and immunohistochemical study of 22 cases. Am J Surg Pathol 1998;22:526-537.

4 Cuttitta F, Carney DN, Mulshine J, et al. Bombesin-like peptides can function as autocrine growth factors in human small cell cancer. Nature 1985;316: 823-826.

5 Rozengurt E. Autocrine loops, signal transduction, and cell cycle abnormalities in the molecular biology of lung cancer. Curr Opin Oncol 1999;11:116-122.

6 Lee JE. Basic helix-loop-helix genes in neural development. Curr Opin Neurobiol 1997;7:13-20.

7 Jan YN, Jan LY. HLH proteins, fly neurogenesis, and vertebrate myogenesis. Cell 1993;75:827-830.

8 Guillemot F, Lo LC, Johnson JE, et al. Mammalian achaete-scute homolog 1 is required for the early development of olfactory and autonomic neurons. Cell 1993;75:463-476.

9 Villares R, Cabrera CV. The achaete-scute gene complex of D. melanogaster: conserved domains in a subset of genes required for neurogenesis and their homology to myc. Cell 1987;50:415-424.

10 Campuzano S, Modolell J. Patterning of the Drosophila nervous system: the achaete-scute gene complex. Trends Genet 1992;8:202-208.

11 Johnson JE, Birren SJ, Anderson DJ. Two rat homologues of Drosophila achaete-scute specifically expressed in neuronal precursors. Nature 1990;346: 858-861.

12 Ball DW, Azzoli CG, Baylin SB, et al. Identification of a human achaete-scute homolog highly expressed in neuroendocrine tumors. Proc Natl Acad Sci USA 1993;90:5648-5652.

13 Lo LC, Johnson JE, Wuenschell CW, et al. Mammalian achaete-scute homolog 1 is transiently expressed by spatially restricted subsets of early neuroepithelial and neural crest cells. Genes Dev 1991;5:1524-1537.

14 Borges M, Linnoila RI, van de Velde HJK, et al. An achaete-scute homologue essential for neuroendocrine differentiation in the lung. Nature 1997;386:852-855.

15 Ito T, Udaka N, Yazawa T, et al. Basic helix-loop-helix transcription factors regulate the neuroendocrine differentiation of fetal mouse pulmonary epithelium. Development 2000;127:3913-3921.

16 Lanigan TM, DeRaad SK, Russo AF. Requirement of the MASH-1 transcription factor for neuroendocrine differentiation of thyroid $\mathrm{C}$ cells. J Neurobiol 1998;34:126-134.

17 Huber K, Bruhl B, Guillemot F, et al. Development of chromaffin cells depends on MASH1 function. Development 2002;129:4729-4738.

18 Chen H, Thiagalingam A, Chopra H, et al. Conservation of the Drosophila lateral inhibition pathway in human lung cancer: a hairy-related protein (HES-1) directly represses achaete-scute homolog-1 expression. Proc Natl Acad Sci USA 1997;94:5355-5360.
19 Chen H, Biel MA, Borges MW, et al. Tissue-specific expression of human achaete-scute homologue-1 in neuroendocrine tumors: transcriptional regulation by dual inhibitory regions. Cell Growth Differ 1997;8:677-686.

20 Chen H, Carson-Walter EB, Baylin SB, et al. Differentiation of medullary thyroid cancer by C-Raf-1 silences expression of the neural transcription factor human achaete-scute homolog-1. Surgery 1996;120:168-173.

21 Persson P, Jogi A, Grynfeld A, et al. HASH-1 and E2-2 are expressed in human neuroblastoma cells and form a functional complex. Biochem Biophys Res Commun 2000;274:22-31.

22 Soderholm H, Ortoft E, Johansson I, et al. Human achaete-scute homologue 1 (HASH1) is downregulated in differentiating neuroblastoma cells. Biochem Biophys Res Commun 1999;256:557-563.

23 Rostomily RC, Bermingham-McDonogh O, Berger MS, et al. Expression of neurogenic basic helix-loop-helix genes in primitive neuroectodermal tumors. Cancer Res 1997;57:3526-3531.

24 Travis WD, Colby TV, Corrin B, et al. (in collaboration with Sobin $\mathrm{LH}$ and pathologists from 14 countries). The World Health Organization Histological Typing of Lung and Pleural Tumors, 3rd edn. Springer: Tokyo, 1999.

25 Kerstens HM, Poddighe PJ, Hanselaar AG. A novel in situ hybridization signal amplification method based on the deposition of biotinylated tyramine. J Histochem Cytochem 1995;43:347-352.

26 Jiang SX, Kameya T, Sato U, et al. Bcl-2 protein expression in lung cancer and close correlation with neuroendocrine differentiation. Am J Pathol 1996;148: 837-846.

27 Rindi G, Buffa R, Sessa F, et al. Chromogranin A, B and $\mathrm{C}$ immunoreactivities of mammalian endocrine cells. Distribution, distinction from costored hormones/prehormones and relationship with the argyrophil component of secretory granules. Histochemistry 1986;85:19-28.

28 Huttner WB, Gerdes HH, Rosa P. The granin (chromogranin/secretogranin) family. Trends Biochem Sci 1991;16:27-30.

29 Rosa P, Gerdes HH. The granin protein family: markers for neuroendocrine cells and tools for the diagnosis of neuroendocrine tumors. J Endocrinol Invest 1994;17: 207-225.

30 Feldman SA, Eiden LE. The chromogranins: their roles in secretion from neuroendocrine cells and as markers for neuroendocrine neoplasia. Endocr Pathol 2003;14: $3-23$.

31 Johnson JE, Zimmerman K, Saito T, et al. Induction and repression of mammalian achaete-scute homologue (MASH) gene expression during neuronal differentiation of P19 embryonal carcinoma cells. Development 1992;114:75-87. 